\title{
Neural circuits for sexually dimorphic and sexually divergent behaviors in C. elegans
}

\author{
L. René García and Douglas S. Portman
}

L. Rene Garcia, Ph.D.

Department of Biology, Howard Hughes Medical Institute, Texas A\&M University

3258 TAMU, College Station, TX 77843-3258

E-mail: rgarcia@mail.bio.tamu.edu

Tel: (979) 845-2989

Fax: (979) 845-2891

Douglas S. Portman, Ph.D.

Center for Neural Development and Disease, University of Rochester

601 Elmwood Ave., Box 645, Rochester NY 14642

E-mail: douglas.portman@rochester.edu

Tel: (585) 275-7414

Fax: (585) 275-1947

\begin{abstract}
Increasing interest in sex differences in C. elegans neurobiology is resulting from several advances, including the completion of the male tail connectome and the surprising discovery of two "new" neurons in the the male head. In this species, sex-specific circuits in the hermaphrodite and male control reproductive behaviors such as egg-laying and copulation, respectively. Studies of these systems are revealing interesting similarities and contrasts, particularly in the mechanisms by which nutritional status influences reproductive behaviors. Other studies have highlighted the importance of sexual modulation of shared neurons and circuits in optimizing behavioral strategies. Together, these findings indicate that $C$. elegans uses intertwined, distributed sex differences in circuit structure and function to implement sexspecific as well as sexually divergent, shared behaviors.
\end{abstract}




\section{INTRODUCTION}

Though the nematode $C$. elegans has been the subject of extraordinary experimental efforts, the vast majority of this work has focused on just one sex, the hermaphrodite (a selffertile somatic female). However, increasing interest is being paid to the C. elegans male. A key driver of this is the recent completion of the adult male tail connectome, which has led to important new insights into the neural and genetic basis of sexually dimorphic behavior [1]. Several comprehensive reviews provide overviews of male development and function [2-4]. Here, we review recent advances in understanding the development and function of sex-specific circuits, as well as work exploring the sexual modulation of anatomically shared circuits.

A well-characterized sex-determination pathway links $C$. elegans chromosomal sex to the transcription factor TRA-1A, which specifies nearly all sex differences in the soma (see Box 1). In the nervous system, the most obvious aspect of sexual differentiation is in anatomical composition: adult hermaphrodites possess eight sex-specific neurons, while adult males have 91, all of which are integrated into a network of 294 shared neurons [5]. These sex-specific neurons, found largely in the midbody and tail, contribute to sexually dimorphic circuits that subserve male copulatory behavior and hermaphrodite egg-laying. In addition, recent work has highlighted the importance of sex-specific "tuning” of shared neurons and circuits for both sexspecific and sexually modulated behaviors.

\section{Sex-specific genital circuitry and reproductive behavioral dimorphism}


The reproductive circuitry of both sexes is optimized to disperse progeny in food-rich environments. When food is scarce, hermaphrodites retain eggs in their uterus [6], and males do not sustain copulation [7]. However, in abundant food, hermaphrodites regulate the frequency of their egg-laying behavior to widely distribute their eggs [8], and males roam to copulate promiscuously [9]. In both sexes, motor regulation is executed by sex-specific muscles derived from the same progenitor cell [10].

\section{Egg-laying behavior}

The hermaphrodite egg-laying muscles are derived from the embryonic M cell (Fig. 1A, B). They consist of 8 uterine muscles, which squeeze the eggs from the uterus, and 8 vulva muscles, which open the vulval slit (Fig. 1C). These electrically connected muscles are stimulated by the cholinergic VC and serotonergic HSN neurons, both of which are present only in hermaphrodites (Fig. 1D) [11]. The egg-laying circuitry is regulated to allow stochastic dispersion of eggs, presumably to reduce local overcrowding and food competition.

Egg dispersion is accomplished by coupling egg-laying with locomotion. A well-fed hermaphrodite alternates between inactive and active egg-laying states, during which she intermittently lays eggs [8] (Fig. 2A). The HSNs modulate the transition between these two states. During the inactive state, the HSNs are down-modulated by the G-protein receptors EGL47 and EGL-6, the G-protein $\mathrm{G}_{0}$ and the hyper-polarizing IRK-1 $\mathrm{K}^{+}$channel $[12,13]$. When the HSNs are active, their secretions synergize with acetylcholine from the VC neurons to adjust the frequency of egg-laying muscle contractions. Genetic analyses indicate that the HSNs' stochastic 
activity pattern is regulated by hyperpolarizing chloride conductance through the CLH-3 chloride channels [14•]. Analogous to the HSNs, VC secretions are negatively regulated by the small conductance calcium-activated $\mathrm{K}^{+}$channel KCNL-2; experimentally, channel over-expression causes abnormal egg-laying patterns, mimicking removal of the VCs [15].

VC activity patterns are influenced by locomotion-coupled body bends. Coordinated body wall muscle and uterine/vulval muscle contractions likely force the expulsion of eggs. The VCs are hypothesized to sense postural changes and subsequently secrete acetylcholine, which hypopolarizes the vulva muscles. However, hyperpolarizing current from UNC-103 and other $\mathrm{K}^{+}$ channels maintain the muscles at a sub-firing threshold, keeping them from prematurely contracting. This allows the egg-laying machinery to contract only when the VC-potentiated muscles are co-stimulated by the HSNs (Fig. 2A) [16].

\section{Copulation behavior}

As in the hermaphrodite, the male sex-specific muscles are derived from the M lineage

(Fig. 1A, B). However, the male additionally repurposes and incorporates a shared muscle into his reproductive circuitry. In hermaphrodites and larval males, the anal depressor muscle is used for fecal expulsion. However, during development, the sex-determination pathway and signaling from the adjacent M-lineage cells cause the anal depressor to disassemble its dorsal-ventral sarcomere and form a new anterior-posterior sarcomere over the sex muscles (Fig. 1B) [17••]. This morphological transformation facilitates intromission [18]. The intromission circuitry shares similar signaling molecules as the egg-laying circuit, but produces different outputs $[19,20]$. To 
intromit his copulatory spicules into his mate, the male uses high frequency protractor muscle contractions to rhythmically thrust his spicules at the vulva. These muscles are excited via electrical connections with the remodeled anal depressor and the gubernacular and oblique muscles, which in turn are stimulated by the glutamatergic and cholinergic PCA, PCB and PCC neurons [1] (Fig. 1C\&D).

C. elegans hermaphrodites prefer self-fertilization over outcrossing. Recent work has shown that copulation can be physically harmful, damaging the hermaphrodite's cuticle [21], and even causing death $[22 \bullet \cdot, 23 \bullet \bullet, 24]$. N2 hermaphrodites favor self-reproduction and use their chemosensory neurons to sense males and resist copulation [25]. In response, the male persistently thrusts his spicules to breach the reticent hermaphrodite's vulva. The PCA and PCB sensory neurons sense structural features of the vulval lips, and stimulate each other and the spicule associated muscles (Fig. 2B). The motor pattern repeats until the spicules penetrate, the male is dislodged, or repeated attempts become futile.

Similar to egg-laying behavior, the male’s circuitry uses inhibitory dopamine signaling to dampen motor patterns [26]; however, this occurs when intromission attempts become futile (Fig 2C). The PCA neurons are synapsed onto the glutamatergic chemosensory-interneuron HOA and the dopaminergic ray neurons. Initially, HOA senses the vulva and the dopaminergic ray neurons sense the hermaphrodite cuticle. When penetration attempts initiate, PCA activity is initially moderate; however, its activity increases if attempts are prolonged [18]. At a threshold, PCA neurons are hypothesized to hyper-polarize HOA via the AVR-14 glutamate-gated chloride channel and also stimulate dopamine secretion from the ray neurons. Dopamine then activates 
the DOP-2 and DOP-3 G-protein receptors on the HOA and PCB neurons. These neurons are electrically coupled via UNC-7 and UNC-9 innexins. Dopamine signaling increases innexin conductance, allowing hyperpolarizing current from HOA to flow into and attenuate PCB. This increases the probability that the male will move off the vulva and search for a more receptive mate $[27 \bullet \bullet]$.

\section{Sexual modulation of shared neurons and circuits}

The circuits that enable sex-specific egg-laying and copulation are interconnected with shared neurons and circuits to allow coordinated behavior. Several studies have identified sexually "tuned” features of these shared components, and shown that they are necessary for a variety of sex differences in behavior.

Sex-specific control of motor behavior

Locomotion is the output of most C. elegans behaviors. However, the relationship between male mating, which requires sustained reverse locomotion ("response” behavior), and sex-common motor circuits are poorly understood. Recent work has found that backward locomotion occurs through activation of AVA (a sex-shared reverse command interneuron) by the male-specific PVY/PVX interneurons [28]. These in turn receive direct input from the malespecific tail ray sensilla. 
Other shared components of the motor system also have sexually dimorphic properties. For example, ablation of DVA, a sex-shared mechanosensor that regulates body posture [29], has little effect on hermaphrodite locomotion, but causes motor defects in males [30]. This might indicate that DVA inhibits maladaptive sex differences generated by other components of the male nervous system. DVA secretes the oxytocin/vasopressin ortholog nematocin, which promotes sex-specific behaviors [30]; however, DVA's role in locomotion appears independent of this.

The sexes also differ in baseline locomotion: males display greater amplitude of the body wave as well as body-bend frequency. These features emerge from complex interactions between body mechanics, neuromuscular physiology, and sex-specific properties of the nervous system [31•]. In particular, sex-specific properties of shared sensory neurons control sex differences in body-bend frequency, highlighting the importance of sexual "tuning” of shared sensory function.

Sex differences in shared sensory function

Though the male tail is richly endowed with sensory structures, most sensory input is transmitted through sex-shared sensilla in the head. These organs' anatomy is nearly identical between the sexes. However, multiple studies have identified functional sex differences in sensory function. Sexually dimorphic features of shared neurons, including AWA, AWC, and ASK, have been implicated in olfaction and sex pheromone response [32-34]. Sex differences in shared circuits are also important for behavioral prioritization. Recent work found that regulation of the food chemoreceptor ODR-10 is important for the choice between feeding and exploration 
[35••]. In hermaphrodites, high expression of odr-10 in the AWA chemosensory neuron allows efficient food detection. In males, odr-10 expression is low, making animals prone to leave food and search for mates [35]. Manipulating tra-1 activity (and therefore sexual "state") in this single neuron pair is sufficient to alter the choice between feeding and exploration.

Chemosensory plasticity also differs by sex in C. elegans. $\mathrm{NaCl}$ is an attractive cue in both sexes, but hermaphrodites learn to avoid $\mathrm{NaCl}$ when it is paired with the aversive stimulus of starvation [36]. Interestingly, this is true for males only if hermaphrodites are absent during conditioning [37]. Otherwise, hermaphrodite cues block starvation-induced $\mathrm{NaCl}$ aversion, suggesting that males prioritize the positive association between salt and pheromone. This "sexual conditioning" depends on the male state of the nervous system and may allow animals to efficiently find mates in suboptimal environments [37].

Sex-specific chemical signaling

Outside of sex pheromones, little is known about sex-specific communication in $C$. elegans. Recent work has revealed that the male-specific CEM head sensory neurons secrete extracellular vesicles (ECVs) that contain multiple signaling components [38,39••]. Interestingly, exposure of males to ECVs promotes copulatory behaviors. The mechanisms by which ECVs are generated and by which they signal remain an area of active study.

\section{Development of sex differences in the nervous system}


Until recently, dogma held that adult males possess 383 neurons, 89 of which are malespecific. Remarkably, in the course of studying a gene important for male exploratory behavior, Sammut et al. [40••] discovered two "new" head neurons, the MCMs (mystery cells of the male). These connect male-specific inputs from the tail to head navigation circuitry and are necessary for NaCl-pheromone sexual conditioning. Surprisingly, the MCMs develop from a male-specific division of the fully-differentiated, sex-shared AMso amphid sheath glia during late larval development. This event is controlled cell-autonomously by the sex-determination pathway, as genetic sex-reversal of the AMso glia generates MCMs in hermaphrodites and prevent their development in males [40••].

Aside from the sex-specific deaths of the HSN and CEM neurons [41-43] and the neurogenesis of the MCMs [40••], relatively little is known about the developmental mechanisms that bring about sex differences in nervous system structure. Recently, Hox- and TALE-class transcription factors have been shown to be important for male-specific neurogenesis of the CA and CP neurons in the ventral cord [44]. Additionally, VEGF signaling has been shown to interact with plexin and netrin signaling in the migration of male tail sensilla [45]. However, much remains to be learned about the mechanisms that couple tra-1 to the alterations in lineage and cell fate that build sex-specific and sexually "tuned" circuits.

\section{CONCLUSION}

The study of sex differences in neural development and function in C. elegans is uniquely advantaged by the nearly complete knowledge of the connectomes of both sexes. In some cases, 
downstream effectors of sex determination have been identified, and the mechanisms by which they canalize developmental programs are understood. Studies of the neural logic of sexually dimorphic circuitry has revealed interesting parallels and differences between the sexes, and has shed important light on the ways by which neural circuits optimize reproductive strategies. However, much remains to be learned about the nature of sex differences in the nervous system and the behaviors they regulate. Both of these problems will be informed by new technologies in nervous system imaging and gene expression profiling, but more traditional approaches, including forward-genetic screens, still hold great potential. With continued progress on the mechanisms that control sex differences in nervous system development and physiology, either by gross alterations or subtle adjustments, the nematode C. elegans affords the opportunity to understand how a single genetic signal implements naturally occurring, coordinated variation in nervous system structure, function and behavior.

\section{ACKNOWLEDGEMENTS}

LRG is funded by the Howard Hughes Medical Institute. DSP is funded by the National Institutes of Health (R01 GM108885) and the National Science Foundation (IOS 1353075).

\section{FIGURE LEGENDS}

Figure 1. Egg-laying and male copulatory anatomy. 
(A) Top panel, egg-laying anatomy. Green circles: VC neurons. Blue circle: HSN neuron. Red rectangles: vulva and uterine muscles. Bottom panel, the male copulation musculature. Red rectangles: copulation muscles.

(B) Top panel, developmental lineage of the hermaphrodite and male $\mathrm{M}$ cell. The right bar shows the relative larval stages that the cells divide. Hermaphrodite lineage: u1, uterine muscle 1; u2, uterine muscle 2; v1, vulva muscle 1; v2, vulva muscle 2. vp, ventral protractor; dp, dorsal protractor; dg, diagonal muscle. Male lineage: vr, ventral retractor; dr, dorsal retractor; ao, anterior oblique; po posterior oblique; ge, gubernaculum erector; gr, gubernaculum retractor; ol, outer longitudinal; il, inner longitudinal; cl, caudal longitudinal; c, coelomocyte. Bottom panel, remodeling of the male anal depressor. Blue lines: actin-myosin contractile filaments.

(C) Top panel, egg-laying circuit components around the vulval-uterine region. Bottom panel, male copulation circuit components. Green circles: one set of the bilateral cholinergic PCB and PCC post-cloacal sensilla neurons and the SPC proprioceptive neurons. Pink circle: one set of the glutamatergic PCA post-cloacal sensilla neuron. The HOA neuron is a single glutamatergic neuron located on the left side of the male.

(D) Top panel, egg-laying circuitry. Bottom panel, intromission circuitry. Bars represent electrical connections; arrows represent chemical connections.

\section{Figure 2. Behavioral regulation of hermaphrodite egg-laying and male spicule intromission attempts.}

(A) Left panel, inactive and active phases of egg-laying behavior; right panel, molecular interactions that occur during active egg-laying events. 
(B) Left panel, male tail when it first contacts the vulva. Right panel, cellular and molecular interactions that occur during the initiation of intromission attempts. Solid green circle: the active PCB cholinergic neuron. Solid pink circle: active HOA glutamatergic neuron. Solid red square: rhythmically contracting sex muscles. Graded pink circle represents moderate PCA activity. Graded purple circle: moderate dopaminergic ray neuronal activity.

(C) Left panel cartoon, the male tail moving off the vulva after futile intromission attempts. Right panel, cellular and molecular interactions after prolonged intromission attempts. Graded green circle: hyper-polarized PCB cholinergic neuron. Graded pink circle: hyper-polarized HOA glutamatergic neuron. Graded red square: reduced and erratic contracting sex muscles. Solid pink circle: increased PCA activity. Solid purple circle: increased dopaminergic ray neuronal activity.

\section{BOX 1: Sex determination in C. elegans}

C. elegans features a chromosomal sex-determination system in which X chromosome dosage sets into motion a genetic cascade that controls both dosage compensation and sexual differentiation [46]. This cascade activates the transcription factor TRA-1A in XX animals (hermaphrodites) and represses it in XO males. tra-1 is the master regulator of somatic sex, being both necessary and sufficient for essentially all sexual differentiation the soma. Multiple targets act downstream of tra-1 to implement sex differences in neurogenesis, cell fate, and neurophysiology. Among these are the DM-family genes mab-3, mab-23, and $d m d-3$, which are important for the development and patterning of the male-specific ray sensilla as well as nonneural structures [47-50]. tra-1 also regulates the cell death pathway to control the sex-specific 
survival of the HSN and CEM neurons [41-43]. However, tra-1 almost certainly has additional, yet-unidentified targets important for other sex differences in neural development and function. 


\section{REFERENCES}

1. Jarrell TA, Wang Y, Bloniarz AE, Brittin CA, Xu M, Thomson JN, Albertson DG, Hall DH, Emmons SW: The connectome of a decision-making neural network. Science 2012, 337:437-444.

2. Barr MM, Garcia LR: Male mating behavior. WormBook 2006:1-11.

3. Emmons SW: The development of sexual dimorphism: studies of the Caenorhabditis elegans male. Wiley Interdiscip Rev Dev Biol 2014, 3:239-262.

4. Fagan KA, Portman DS: Sexual modulation of neural circuits and behavior in Caenorhabditis elegans. Semin Cell Dev Biol 2014, 33C:3-9.

5. Portman D: Genetic Control of Sex Differences in C. elegans Neurobiology and Behavior. Adv Genet 2007, 59:1-37.

6. Trent C: Genetic and behavioral studies of the egg-laying system of Caenorhabditis elegans. Edited by. Cambridge: Massachusetts Institute of Technology; 1982. vol Ph.D.]

7. Zhang L, Gualberto DG, Guo X, Correa P, Jee C, Garcia LR: TMC-1 attenuates C. elegans development and sexual behaviour in a chemically defined food environment. Nat Commun 2015, 6.

8. Waggoner LE, Zhou GT, Schafer RW, Schafer WR: Control of alternative behavioral states by serotonin in Caenorhabditis elegans. Neuron 1998, 21:203-214.

9. Lipton J, Kleemann G, Ghosh R, Lints R, Emmons SW: Mate searching in Caenorhabditis elegans: a genetic model for sex drive in a simple invertebrate. J Neurosci 2004, 24:7427-7434.

10. Sulston JE, Albertson DG, Thomson JN: The Caenorhabditis elegans male: postembryonic development of nongonadal structures. Dev Biol 1980, 78:542-576.

11. White JG, Southgate E, Thomson JN, Brenner S: The structure of the nervous system of the nematode Caenorhabditis elegans. Phil Trans R Soc Lond B 1986, 314:1-340.

12. Moresco JJ, Koelle MR: Activation of EGL-47, a Gao-coupled receptor, inhibits function of hermaphrodite-specific motor neurons to regulate Caenorhabditis elegans egglaying behavior. J Neurosci 2004, 24:8522-8530.

13. Emtage L, Aziz-Zaman S, Padovan-Merhar O, Horvitz HR, Fang-Yen C, Ringstad N: IRK-1 potassium channels mediate peptidergic inhibition of Caenorhabditis elegans serotonin neurons via a Go signaling pathway. J Neurosci 2012, 32:16285-16295. 
- 14. Branicky R, Miyazaki H, Strange K, Schafer WR: The voltage-gated anion channels encoded by clh-3 regulate egg laying in $C$. elegans by modulating motor neuron excitability. $J$ Neurosci 2014, 34:764-775.

This study shows how the C. elegans homolog of the CLC-2 hyperpolarization-activated, inwardrectifying chloride channel regulates the hermaphrodite serotonergic HSN activity pattern.

15. Chotoo CK, Silverman GA, Devor DC, Luke CJ: A small conductance calcium-activated $\mathrm{K}+$ channel in $\mathrm{C}$. elegans, $\mathrm{KCNL}-2$, plays a role in the regulation of the rate of egglaying. PLOS ONE 2013, 8:e75869.

16. Collins KM, Koelle MR: Postsynaptic ERG potassium channels limit muscle excitability to alow distinct egg-laying behavior states in Caenorhabditis elegans. J Neurosci 2013, 33:761-775.

•• 17. Chen X, García LR: Developmental alterations of the C. elegans male anal depressor morphology and function require sex-specific cell autonomous and cell nonautonomous interactions. Dev Biol 2015, 398:24-43.

During late-stage development, the larval male morphologically and functionally alters a sexshare defecation muscle to repurpose it for copulation. The study details how muscle remodeling is regulated by both cell-autonomous sex-determination regulation and cellcell communication with adjacent developing sex-specific muscles.

18. LeBoeuf B, Correa P, Jee C, Garcia LR: Caenorhabditis elegans male sensory-motor neurons and dopaminergic support cells couple ejaculation and post-ejaculatory behaviors. Elife 2014, 3.

19. LeBoeuf B, Garcia LR: Cell excitability necessary for male mating behavior in Caenorhabditis elegans is coordinated by interactions between Big Current and Ether-A-Go-Go family K+ Channels. Genetics 2012, 190:1025-1041.

20. Liu Y, LeBeouf B, Guo X, Correa PA, Gualberto DG, Lints R, Garcia LR: A cholinergicregulated circuit coordinates the maintenance and bi-stable states of a sensorymotor behavior during Caenorhabditis elegans male copulation. PLoS Genet 2011, 7:e1001326.

21. Woodruff GC, Knauss CM, Maugel TK, Haag ES: Mating damages the cuticle of $\boldsymbol{C}$. elegans hermaphrodites. PLOS ONE 2014, 9:e104456.

•• 22. Maures TJ, Booth LN, Benayoun BA, Izrayelit Y, Schroeder FC, Brunet A: Males shorten the life span of $\boldsymbol{C}$. elegans hermaphrodites via secreted compounds. Science 2014, 343:541-544.

This paper demonstrates that diffusible substances secreted by males will reduce the lifespan of the hermaphrodite. Mating does not even have to occur; exposure to male-conditioned media is sufficient to reduce longevity. 
•• 23. Shi C, Murphy CT: Mating induces shrinking and death in Caenorhabditis mothers. Science 2014, 343:536-540.

This paper demonstrates that male sperm, introduced into the hermaphrodite via copulation, causes tissue damage such as germline shrinkage, osmotic stress susceptibility and decreased marked lifespan.

24. Ting JJ, Woodruff GC, Leung G, Shin N-R, Cutter AD, Haag ES: Intense sperm-mediated sexual conflict promotes reproductive isolation in Caenorhabditis nematodes. PLoS Biol 2014, 12:e1001915.

25. Bahrami AK, Zhang Y: When females produce sperm: Genetics of C. elegans hermaphrodite reproductive choice. G3 2013, 3:1851-1859.

26. Schafer WR, Kenyon CJ: A calcium-channel homologue required for adaptation to dopamine and serotonin in Caenorhabditis elegans. Nature 1995, 375:73-78.

•• 27. Correa PA, Gruninger T, García LR: DOP-2 D2-Like receptor regulates UNC-7 innexins to attenuate recurrent sensory motor neurons during $C$. elegans copulation. J Neurosci 2015, 35:9990-10004.

Although males have a strong drive to copulate, they must have mechanisms to attenuate their behavior if a potential mate is not cooperative to mating. The study details how the male neurocircuitry uses a form of feed-forward inhibition to modulate how long he spends trying to insert his copulatory spicules into a mating-reticent hermaphrodite.

28. Sherlekar AL, Janssen A, Siehr MS, Koo PK, Caflisch L, Boggess M, Lints R: The C. elegans male exercises directional control during mating through cholinergic regulation of sex-shared command interneurons. PLoS One 2013, 8:e60597.

29. Li W, Feng Z, Sternberg P, Xu X: A C. elegans stretch receptor neuron revealed by a mechanosensitive TRP channel homologue. Nature 2006, 440:684-687.

30. Garrison JL, Macosko EZ, Bernstein S, Pokala N, Albrecht DR, Bargmann CI: Oxytocin/vasopressin-related peptides have an ancient role in reproductive behavior. Science 2012, 338:540-543.

31. Mowrey WR, Bennett JR, Portman DS: Distributed Effects of Biological Sex Define SexTypical Motor Behavior in Caenorhabditis elegans. J Neurosci 2014, 34:1579-1591.

Adult males and hermaphrodites differ markedly in the features of their basal sinusoidal locomotion. This paper shows that these apparently simple sex differences emerge from complex interactions between sexually dimorphic body shape and rigidity, neuromuscular physiology, and sensory neuron function.

- 32. Lee K, Portman D: Neural sex modifies the function of a C. elegans sensory circuit. Curr Biol 2007, 17:1858-1863. 
33. Srinivasan J, Kaplan F, Ajredini R, Zachariah C, Alborn H, Teal P, Malik R, Edison A, Sternberg P, Schroeder F: A blend of small molecules regulates both mating and development in Caenorhabditis elegans. Nature 2008, 454:1115-1118.

34. White J, Nicholas T, Gritton J, Truong L, Davidson E, Jorgensen E: The sensory circuitry for sexual attraction in C. elegans males. Curr Biol 2007, 17:1847-1857.

•• 35. Ryan DA, Miller RM, Lee K, Neal SJ, Fagan KA, Sengupta P, Portman DS: Sex, age, and hunger regulate behavioral prioritization through dynamic modulation of chemoreceptor expression. Curr Biol 2014, 24:2509-2517.

Adult males will often (suicidally) leave a food source to search for mates. This work demonstrates that males dynamically alter their food sensitivity by modulating expression of the chemoreceptor odr-10, allowing well-fed adults to generate exploratory behavior by reducing food detection.

36. Saeki S, Yamamoto M, Iino Y: Plasticity of chemotaxis revealed by paired presentation of a chemoattractant and starvation in the nematode Caenorhabditis elegans. $J$ Exp Biol 2001, 204:1757-1764.

37. Sakai N, Iwata R, Yokoi S, Butcher RA, Clardy J, Tomioka M, Iino Y: A sexually conditioned switch of chemosensory behavior in C. elegans. PLoS One 2013, 8:e68676.

38. Wang J, Kaletsky R, Silva M, Williams A, Haas LA, Androwski RJ, Landis JN, Patrick C, Rashid A, Santiago-Martinez D, et al.: Cell-Specific Transcriptional Profiling of Ciliated Sensory Neurons Reveals Regulators of Behavior and Extracellular Vesicle Biogenesis. Curr Biol 2015, 25:3232-3238.

•• 39. Wang J, Silva M, Haas LA, Morsci NS, Nguyen KC, Hall DH, Barr MM: C. elegans ciliated sensory neurons release extracellular vesicles that function in animal communication. Curr Biol 2014, 24:519-525.

This work shows that the CEMs, male-specific neurons in the head, unexpectedly procude extracellular vesicles (ECVs) that are secreted into the environment. ECVs can potentiate male sexual behavior, revealing a novel mechanism for chemical communication.

•• 40. Sammut M, Cook SJ, Nguyen KC, Felton T, Hall DH, Emmons SW, Poole RJ, Barrios A: Glia-derived neurons are required for sex-specific learning in C. elegans. Nature 2015, 526:385-390.

This paper identifies a previously unknown pair of neurons in the male head, the MCMs, which eluded researchers for decades. Remarkably, the MCMs develop through the malespecific division of a fully differentiated head glial cell, and are required for males to integrate diverse signals to generate behavioral plasticity. 
41. Conradt B, Horvitz HR: The TRA-1A sex determination protein of C. elegans regulates sexually dimorphic cell deaths by repressing the egl-1 cell death activator gene. Cell 1999, 98:317-327.

42. Peden E, Kimberly E, Gengyo-Ando K, Mitani S, Xue D: Control of sex-specific apoptosis in C. elegans by the BarH homeodomain protein $\mathrm{CEH}-30$ and the transcriptional repressor UNC-37/Groucho. Genes Dev 2007, 21:3195-3207.

43. Schwartz H, Horvitz H: The C. elegans protein CEH-30 protects male-specific neurons from apoptosis independently of the Bcl-2 homolog CED-9. Genes Dev 2007, 21:3181-3194.

44. Kalis AK, Kissiov DU, Kolenbrander ES, Palchick Z, Raghavan S, Tetreault BJ, Williams E, Loer CM, Wolff JR: Patterning of sexually dimorphic neurogenesis in the caenorhabditis elegans ventral cord by Hox and TALE homeodomain transcription factors. Dev Dyn 2014, 243:159-171.

45. Dalpe G, Tarsitano M, Persico MG, Zheng H, Culotti J: C. elegans PVF-1 inhibits permissive UNC-40 signalling through CED-10 GTPase to position the male ray 1 sensillum. Development 2013, 140:4020-4030.

46. Wolff JR, Zarkower D: Somatic sexual differentiation in Caenorhabditis elegans. Curr Top Dev Biol 2008, 83:1-39.

47. Yi W, Ross $\mathrm{JM}$, Zarkower D: $\mathbf{m a b}$-3 is a direct tra-1 target gene regulating diverse aspects of $\boldsymbol{C}$. elegans male sexual development and behavior. Development 2000, 127:4469-4480.

48. Lints R, Emmons SW: Regulation of sex-specific differentiation and mating behavior in C. elegans by a new member of the DM domain transcription factor family. Genes Dev 2002, 16:2390-2402.

49. Siehr MS, Koo PK, Sherlekar AL, Bian X, Bunkers MR, Miller RM, Portman DS, Lints R: Multiple doublesex-related genes specify critical cell fates in a $\mathrm{C}$. elegans male neural circuit. PLoS One 2011, 6:e26811.

50. Mason D, Rabinowitz J, Portman D: dmd-3, a doublesex-related gene regulated by tra-1, governs sex-specific morphogenesis in C. elegans. Development 2008, 135:2373-2382. 

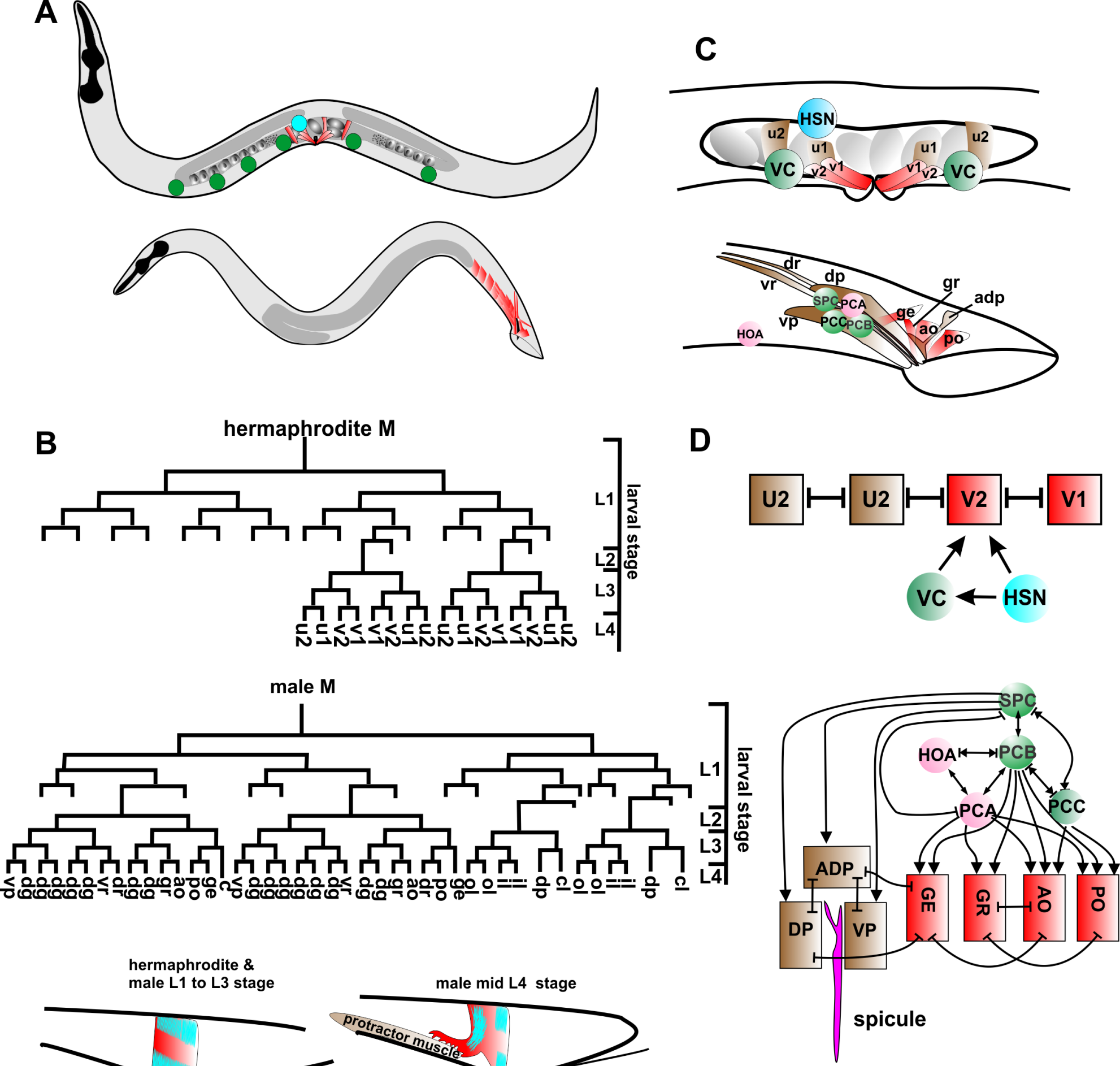

Figure 1

hermaphrodite \&

male L1 to L3 stage

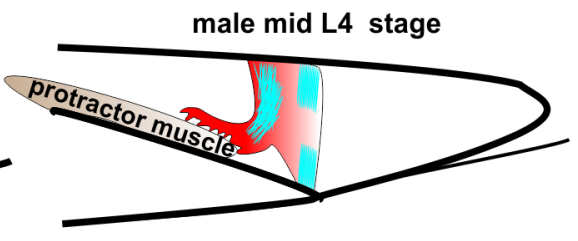

male late L4 stage

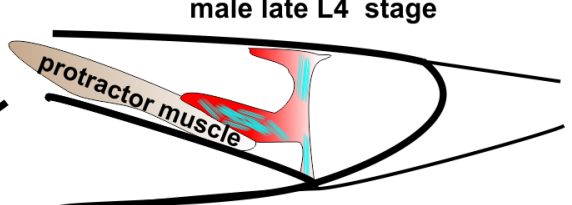

male adult stage

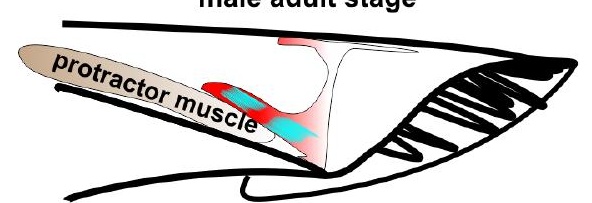


active phase
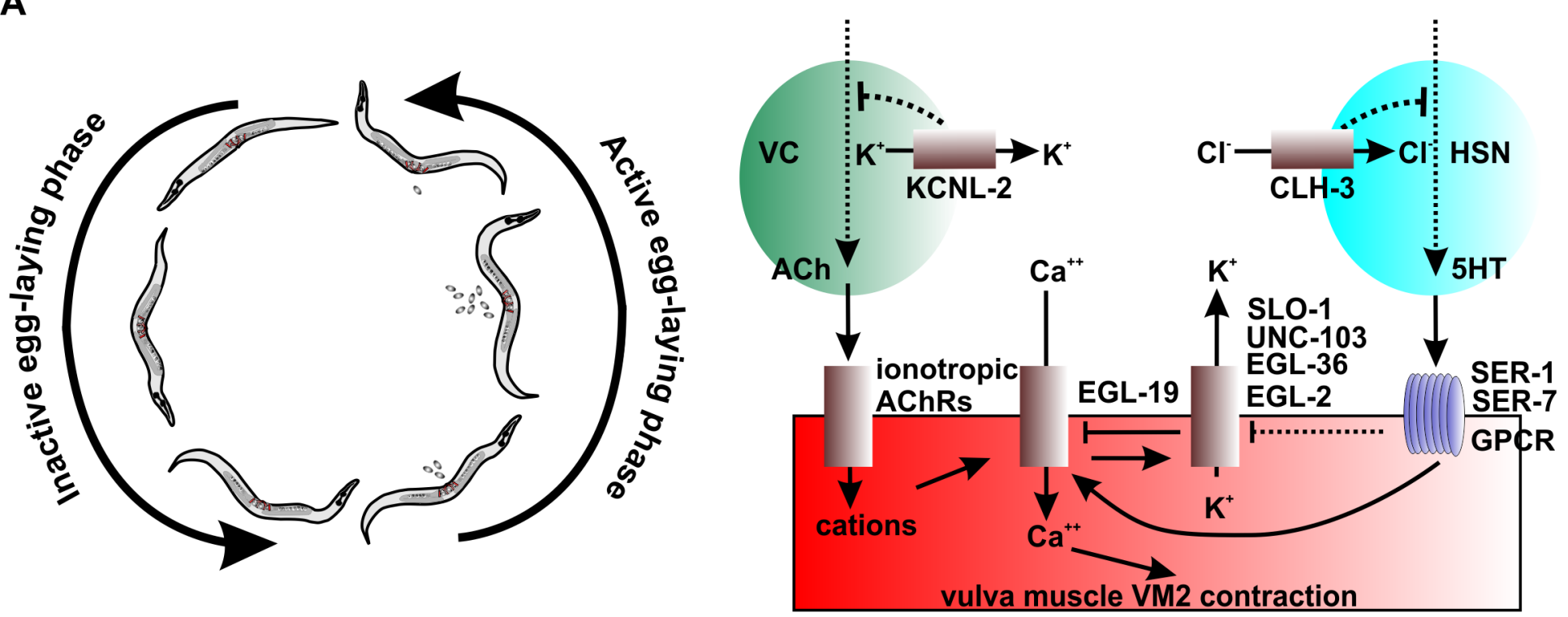

B

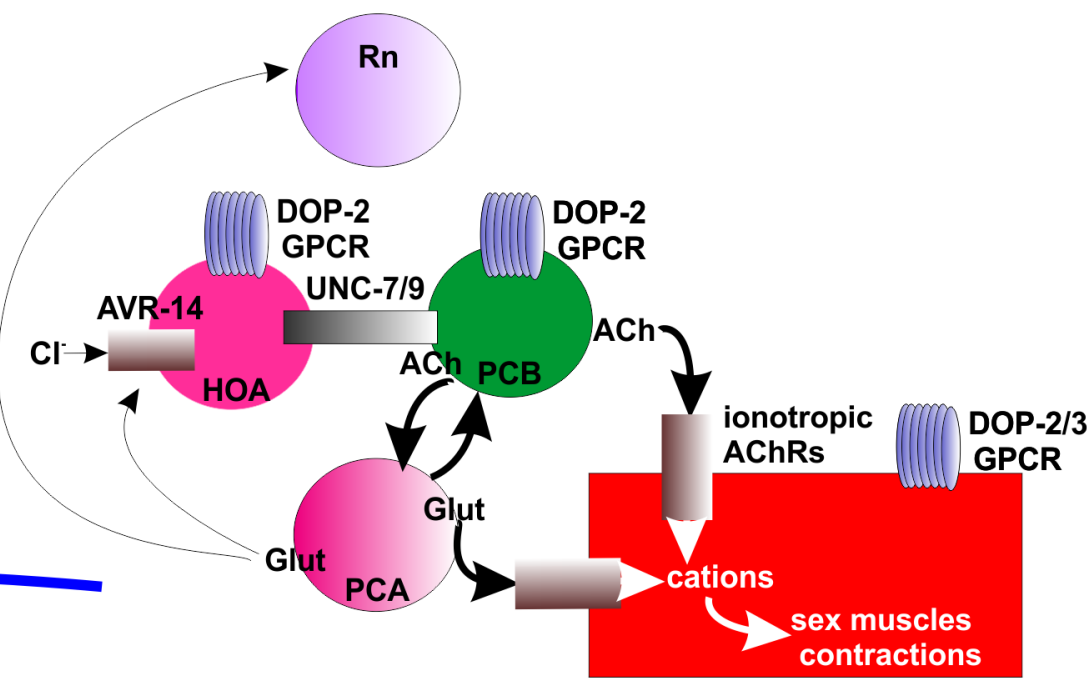

After prolonged unsuccessful intromission attempts

Initiation of spicule intromission attempts

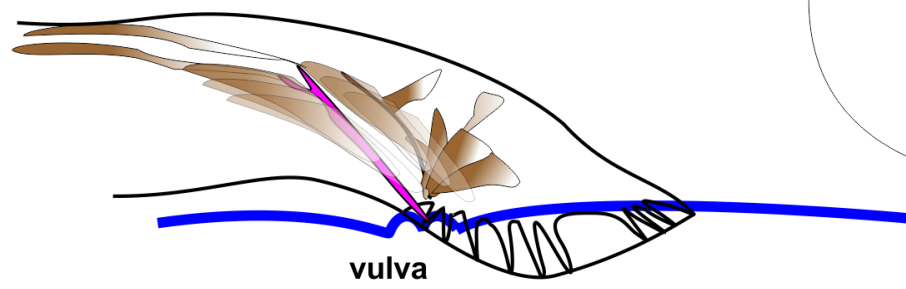

contractions

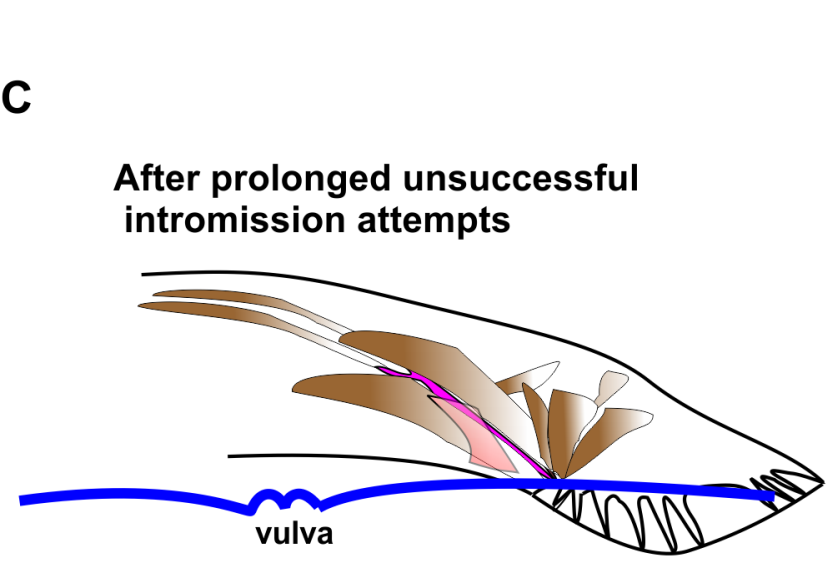

Figure 2

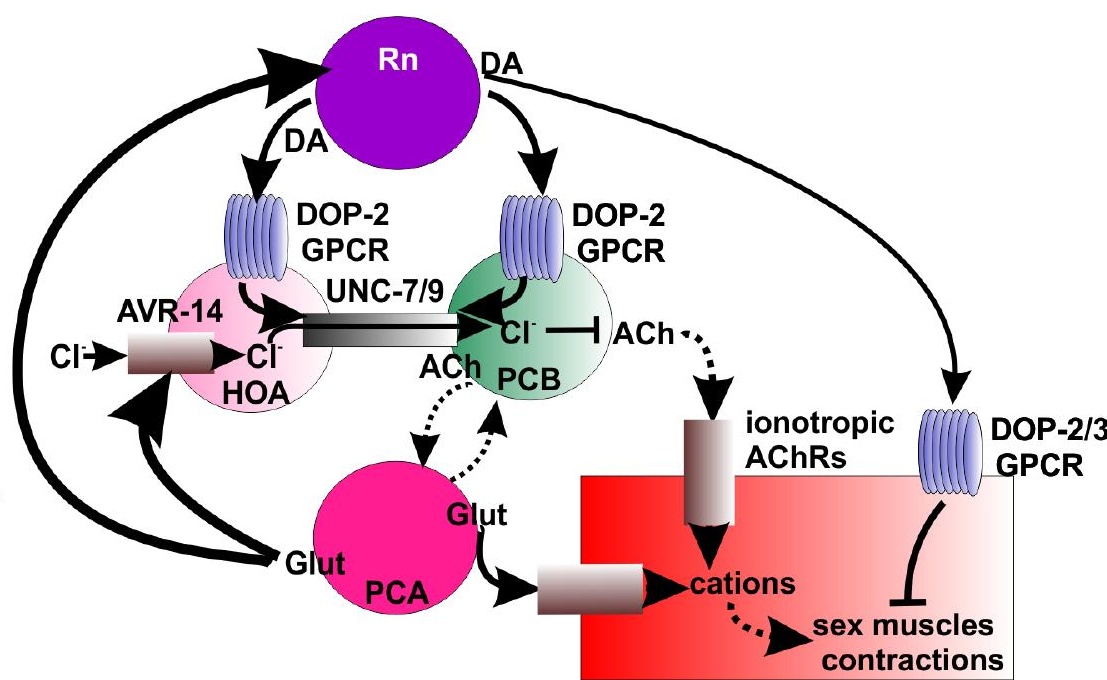

